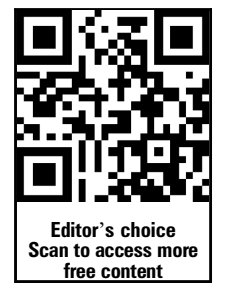

- Additional material is published online only. To view please visit the journal online (http://dx.doi.org/10.1136/ bjsports-2013-093398).

${ }^{1}$ School of Allied Health, La Trobe University, Bundoora, Victoria, Australia

${ }^{2}$ Epworth Healthcare,

Richmond, Victoria, Australia

Correspondence to Dr Clare L Ardern, School of Allied Health, Faculty of Health Sciences, La Trobe University, Bundoora, VIC 3086, Australia; c.ardern@latrobe.edu.au

Accepted 5 August 2014 Published Online First 25 August 2014

\title{
Fifty-five per cent return to competitive sport following anterior cruciate ligament reconstruction surgery: an updated systematic review and meta- analysis including aspects of physical functioning and contextual factors
}

\author{
Clare L Ardern, ${ }^{1}$ Nicholas F Taylor, ${ }^{1}$ Julian A Feller, ${ }^{1,2}$ Kate E Webster $^{1}$
}

\begin{abstract}
Background The aim of this study was to update our original systematic review of return to sport rates following anterior cruciate ligament $(A C L)$ reconstruction surgery.

Method Electronic databases were searched from April 2010 to November 2013 for articles reporting the number of patients returning to sport following $\mathrm{ACL}$ reconstruction surgery. Return to sport rates, physical functioning and contextual data were extracted and combined using random-effects meta-analyses. Data from the original review (articles published up to April 2010) were combined with data from the updated search.
\end{abstract}

Results Sixty-nine articles, reporting on 7556 participants, were reviewed. On average, $81 \%$ of people returned to any sport, $65 \%$ returned to their preinjury level of sport and $55 \%$ returned to competitive level sport after surgery. Symmetrical hopping performance $(d=0.3)$ and the contextual factors of younger age $(d=-0.3)$, male gender $(O R=1.4)$, playing elite sport $(\mathrm{OR}=2.5)$ and having a positive psychological response $(d=0.3)$ favoured returning to the preinjury level sport. Receiving a hamstring tendon autograft favoured returning to competitive level sport $(\mathrm{OR}=2.4)$, whereas receiving a patellar tendon autograft favoured returning to the preinjury level sport $(O R=1.2)$.

Conclusions Returning to sport varied according to different physical functioning and contextual factors, which could warrant additional emphasis in postoperative rehabilitation programmes to maximise participation.

\section{INTRODUCTION}

Return to participation in sport is an important outcome when evaluating the success of anterior cruciate ligament (ACL) reconstruction surgery. In 2010, we reviewed the orthopaedic sports medicine literature that reported return to sport following ACL reconstruction surgery. ${ }^{1}$ The results of 48 studies and 5770 participants were combined, using meta-analyses. ${ }^{1}$ The meta-analyses highlighted that only approximately two in three individuals returned to their preinjury level of sports participation and less than half returned to competitive level sport. ${ }^{1}$

Only $6^{2-7}$ of the 48 studies in our original review reported return to sport as a primary outcome. The WHO, through the International
Classification of Functioning, Disability, and Health (ICF) ${ }^{8}$ has called for the emphasis of health outcome evaluation to be on an individual's ability to participate in society. Therefore, it follows that greater emphasis should be placed on evaluating participation-based outcomes, such as return to sport, following ACL reconstruction surgery. ${ }^{1}$

Since the original review, the reporting of return to sport outcomes has increased. The addition of further data from studies published after the original review also allows other subgroup analyses to be conducted that may enable a more detailed examination of the influence of key background factors such as age, gender, graft type and sports participation level-termed contextual factors in the ICF-on returning to sport than was previously possible. Contextual factors are critical components of the ICF because these factors may impact on an individual's functioning. Therefore, we also analysed the impact of relevant contextual factors on returning to participation in sport after surgery.

Our updated review had the following aims:

Primary aim

To determine the return to sport rate following ACL reconstruction surgery.

Secondary aims

1. To determine whether improved physical functioning favoured returning to sport following ACL reconstruction.

2. To determine whether specific contextual factors favoured returning to sport following ACL reconstruction.

\section{METHODS}

The Preferred Reporting Items for Systematic Reviews and Meta-Analyses (PRISMA) guidelines were followed when conducting and reporting this updated review and meta-analysis. Consistent with the original review and meta-analyses, the ICF was used as a conceptual framework for reporting the updated review findings.

\section{Literature searching}

The search strategy reported in the original review ${ }^{1}$ was applied to the same electronic databases. These databases were: MEDLINE, CINAHL, EMBASE, PsychInfo, SPORTDiscus, The Cochrane Library, AMED, AMI, AusportMed and PEDro. The Meditext database was not searched as it ceased to exist as of January 2010. Reference lists of relevant 
articles and the ePublication lists of key journals were also manually searched to identify any further articles not identified in the electronic database search. In the original review, database searching was completed from the earliest possible entry to April 2010. In the updated review, the literature was searched from April 2010 to November 2013 (inclusive).

\section{Selection criteria}

Articles were included that reported the number or percentage (or data allowing calculation) of participants returning to sport following ACL reconstruction surgery. Non-English language reports, conference abstracts, and review or clinical commentary articles were excluded. The titles and abstracts of articles retrieved from the electronic database search were screened to determine eligibility for inclusion in the updated review. Where eligibility was unclear from the title or abstract, the article was obtained in full text. Two reviewers applied the selection criteria independently. Discrepancies were resolved via consensus, and a third reviewer was consulted if consensus was not achieved.

\section{Assessment of study quality}

The six-item quality assessment checklist developed for the original review ${ }^{1}$ was used to evaluate risk of bias in articles included in the updated review. It is recommended that researchers develop a checklist or modify an existing one to meet the requirements of the review in addressing key issues of methodological quality, particularly where different research designs are included. ${ }^{9}{ }^{10}$ The six items assessed were: (1) participant selection criteria described, (2) representative population selected, (3) participants' preinjury sports participation level reported, (4) data collected prospectively, (5) demographic data reported (at least gender and point estimate of age) and (6) postoperative sports participation level compared with preinjury sports participation status. Items 1 and 2 were taken from Downs and Black's ${ }^{11}$ Checklist for the Assessment of Methodological Quality of Randomised and Non-Randomised Studies. Item 4 was taken from Slim et al ${ }^{12}$ Methodological Index for Non-Randomised Studies. Items 3, 5 and 6 were developed specifically for this review. The purpose of evaluating the study quality was to identify aspects of study design and methodological quality common to all included articles. Two reviewers assessed included articles for risk of bias independently, with consensus used to resolve discrepancies. For each item, articles were assessed as having fulfilled or not fulfilled the criterion. Articles were not excluded on the basis of potential for bias.

\section{Data extraction and synthesis}

We added data from our original review to data extracted from studies included in the updated review. Data were obtained with the data extraction tool used in the original review, and analysed using StatsDirect (Altrincham, UK). A p value of $<0.05$ was used to denote statistical significance for all analyses. Table 1 lists the variables that were extracted for analysis. The primary variables were return to sport rates; and the secondary variables were measures of physical functioning and contextual factors that may impact on participation in sport.

Pooled dichotomous data were analysed using random-effects proportion meta-analyses (weighted for individual study sample size) and ORs. Pooled continuous data were analysed using random-effects meta-analyses and standardised mean differences (also known as effect sizes). Cohen's guidelines ${ }^{13}$ were used when interpreting the pooled standardised mean differences.
Table 1 Variables extracted for analysis

\begin{tabular}{|c|c|c|}
\hline $\begin{array}{l}\text { Primary } \\
\text { variables }\end{array}$ & ICF participation domain & $\begin{array}{l}\text { Return to any sport } \\
\text { Return to preinjury sport } \\
\text { Return to competitive sport }\end{array}$ \\
\hline \multirow[t]{2}{*}{$\begin{array}{l}\text { Secondary } \\
\text { variables }\end{array}$} & $\begin{array}{l}\text { ICF impairments and activity } \\
\text { domains (physical } \\
\text { functioning) }\end{array}$ & $\begin{array}{l}\text { Objective knee function (IKDC } \\
\text { evaluation) } \\
\text { Subjective knee function (IKDC } \\
\text { form) } \\
\text { Hopping performance (hop } \\
\text { distance) } \\
\text { Sport-specific activity } \\
\text { participation frequency (Marx } \\
\text { Activity Scale) }\end{array}$ \\
\hline & ICF contextual factors & $\begin{array}{l}\text { Person-related factors } \\
\text { Age } \\
\text { Gender } \\
\text { Psychological response } \\
\text { Surgery-related factors } \\
\text { Autograft type (hamstring } \\
\text { tendon vs patellar tendon) } \\
\text { Other factors } \\
\text { Sports performance level (elite } \\
\text { vs non-elite) } \\
\text { Length of follow-up } \\
\text { (<12; } 12-36 ;>36 \text { months) }\end{array}$ \\
\hline
\end{tabular}

To address the primary aim of this review, return to sport proportions for individual studies and pooled estimates were summarised in forest plots; and presented separately for the return to any sport rate, return to preinjury level sport rate and return to competitive sport rate. To address the secondary aims of this review, data were compared between people who did and did not return to sport to determine whether physical functioning and contextual factors favoured returning to sport.

Data were extracted and combined ensuring that data from the same participants were not included twice. If data were reported for the same participants in more than one article, data were extracted from the article that reported a higher return to sport rate. If an article reported return to sport data for the same participants at multiple follow-ups, the highest return to sport rate reported in the article was extracted. The degree of heterogeneity within the results of the included studies was assessed using the $\mathrm{I}^{2}$ index, with a larger score indicating that a greater proportion of the variability in the results could be attributed to heterogeneity. ${ }^{14}$

To examine for differences in return to sport rates between elite and non-elite athletes, athletes who played professional sport, or where it was clearly stated that they participated at the highest possible competitive level for their chosen sport (eg, in college sport, National Collegiate Athletic Association Division I), were identified as elite. To evaluate the influence of graft type, return to sport data for participants who received hamstring tendon grafts were compared with data from participants who received patellar tendon grafts, as these were the most commonly reported graft types. To examine the influence of length of follow-up, return to sport data were grouped for short-term (up to 12 months) medium-term (12-36 months) and long -term (>36 months) follow-up.

\section{RESULTS}

The updated literature search from April 2010 identified 373 potentially eligible articles, of which 336 were immediately excluded based on a review of the title and abstract. 
Thirty-seven articles were obtained in full text, and the selection criteria reapplied. Following this, 16 articles were excluded, adding 21 articles to the updated review (figure 1). The independent reviewers had discrepancies in selection criteria application for three articles. On all occasions, discrepancies were resolved via consensus discussion. Full references and reasons for exclusion are provided in online supplementary appendix A. Therefore, this review is based on data from 69 publications (figure 1), 21 identified in the updated search and 48 from the original review. ${ }^{1}$

Two articles reported return to sport data from the same group of 196 competitive athletes, ${ }^{15}{ }^{16}$ and two articles reported return to sport data from a single cohort of 83 participants. ${ }^{17} 18$ Four articles reported return to sport outcomes at multiple follow-up points. ${ }^{6} 151920$ Adjustments were made to account for this in the meta-analyses, and are described in online supplementary appendix A.

\section{Assessment of study quality}

On all occasions, discrepancies between assessors were resolved via consensus discussion. There were discrepancies between reviewers on 12 articles. Most frequently, the discrepancies were for item 2 (in seven articles), which assessed the representativeness of the study sample. Overall, $87 \%$ of the included articles reported participant selection criteria and $88 \%$ reported demographic data. Seventy-four per cent of the included articles reported a return to preinjury sport rate. However, only $64 \%$ of included articles reported the preinjury sports participation level of participants. Sixty-eight per cent of included articles were prospective studies, and $68 \%$ included a representative sample. Details of the assessment of risk of bias assessment for each article are presented in online supplementary appendix B.

\section{Demographic data}

There were a total of 7556 participants (4892 men, 66\%) evaluated in the 69 articles (see online supplementary table S1). In four articles, participant gender was not reported, ${ }^{21-24}$ and in two articles participant gender was inferred from the sport (American football) participants played before injury. ${ }^{25}$ The mean age at surgery was $25.8(\mathrm{SD}=3.2)$ years. Seven articles did not report participant age. ${ }^{21} 2326-30$ Graft type was reported for 7221 participants; 2427 (34\%) received a hamstring tendon graft, and $4405(61 \%)$ received a patellar tendon graft. Nine articles did not specify graft type. ${ }^{2}{ }^{7} 31-37$ Six articles reported that their patient cohorts included people who had undergone previous ACL surgery (ACL reconstruction or repair), ${ }^{26} 38-42$ and in all of these articles the proportion of patients with a history of ACL surgery was less than one-fifth of the cohort. No articles were included for review that only reported return to sport outcomes for patients after revision or bilateral ACL reconstruction surgery.

\section{Participation: return to sport rate}

A total of 4837 participants (57 studies) had returned to some form of sport (figure 2) following ACL reconstruction surgery, for a pooled return to sport rate of $81 \%$ (95\% CI $74 \%$ to $87 \%$; $\left.\mathrm{I}^{2}=98 \%\right)$. There were 2663 participants (50 studies) who had returned to their preinjury level of sport following surgery (figure 3), for a pooled return to sport rate of $65 \%$ (95\% CI $59 \%$ to $\left.72 \% ; \mathrm{I}^{2}=95 \%\right)$. There were 1338 participants (30 studies) who had returned to competitive sport (figure 4) following surgery, for a pooled return to sport rate of 55\% (95\% CI $46 \%$ to $63 \%$; $\left.\mathrm{I}^{2}=95 \%\right)$.
Impairments of body structure and function and activity limitations

Pooled International Knee Documentation Committee (IKDC) objective classification data from three articles $(n=597)^{15} 3943$ showed that people with 'normal' or 'nearly normal' function had similar odds of returning to their preinjury level sport as people with 'abnormal' or 'severely abnormal' function (OR, $95 \%$ CI 2.3, 0.9 to $6.4, p=0.1$ ). However, people with objective knee function classified as 'normal' (IKDC category A) had greater odds of returning to their preinjury level sport than those with objective knee function classified as 'nearly normal', 'abnormal' or 'severely abnormal' (IKDC categories B, C and D, respectively) (OR, 95\% CI 1.9, 1.1 to $3.5, \mathrm{p}=0.04)$. Of those who had returned to their preinjury level sport, $44 \%$ had objective knee function classified as 'normal'. In comparison, $29 \%$ of those who did not return to their preinjury level sport had knee function classified as 'normal'.

The pooled standardised mean difference for IKDC subjective knee function scores from two articles $(n=272)^{34} 39$ was 0.9 (95\% CI 0.004 to $1.8, \mathrm{p}<0.05)$, indicating that positive subjective rating of knee function favoured returning to the preinjury level sport.

The pooled standardised mean difference for the hop test limb symmetry index from two articles $(n=265)^{4}{ }^{34}$ was 0.3 (95\% CI 0.002 to $0.5, \mathrm{p}<0.05)$, indicating that a more symmetrical hopping performance favoured returning to the preinjury level sport.

The pooled standardised mean difference for frequency of participation in sport-specific activities (Marx Activity Scale) from two articles $(n=131)^{37} 44$ was 3.1 (95\% CI 0.4 to 5.9, $\mathrm{p}<0.01$ ), indicating that more frequent participation in running, cutting, decelerating and pivoting activities favoured returning to the preinjury level sport.

\section{Contextual factors}

Person-related factors

The pooled standardised mean difference for age from two articles $(n=281)^{34} 39$ was $-0.3(95 \%$ CI -0.6 to $-0.1, p<0.01)$, indicating that younger age favoured returning to the preinjury level sport.

Pooled data from 18 articles $(n=1950)$ showed that men had greater odds than women of returning to their preinjury level sport (OR, 95\% CI 1.4, 1.2 to $1.7, \mathrm{p}<0.001)$. Pooled data from 10 articles $(n=918)$ also showed that men had greater odds than women of returning to competitive level sport (OR, 95\% CI $1.7,1.2$ to $2.3, \mathrm{p}<0.002)$. The proportions of men and women returning to sport are shown in table 2 .

The pooled standardised mean difference for fear of reinjury from two articles $(n=156)^{4}{ }^{34}$ was 0.9 (95\% CI 0.6 to 1.3 , $\mathrm{p}<0.001$ ), indicating that lower fear of reinjury favoured returning to the preinjury level sport. The pooled standardised mean difference from two articles $(n=307)^{4} 7$ for psychological readiness to return to sport was 1.0 (95\% CI 0.6 to $1.4, \mathrm{p}<0.001$ ), indicating that greater psychological readiness to return to sport favoured returning to the preinjury level sport.

\section{Surgery-related factors}

Pooled data from 32 articles $(n=4091)$ showed that people who received hamstring tendon grafts had almost twice the odds of returning to any sport compared with those with patellar tendon grafts (OR, 95\% CI 1.7, 1.4 to $2.0, \mathrm{p}<0.001)$. Pooled data from 12 articles $(n=1558)$ also showed that people who received hamstring tendon grafts had more than twice the odds 
Original literature search

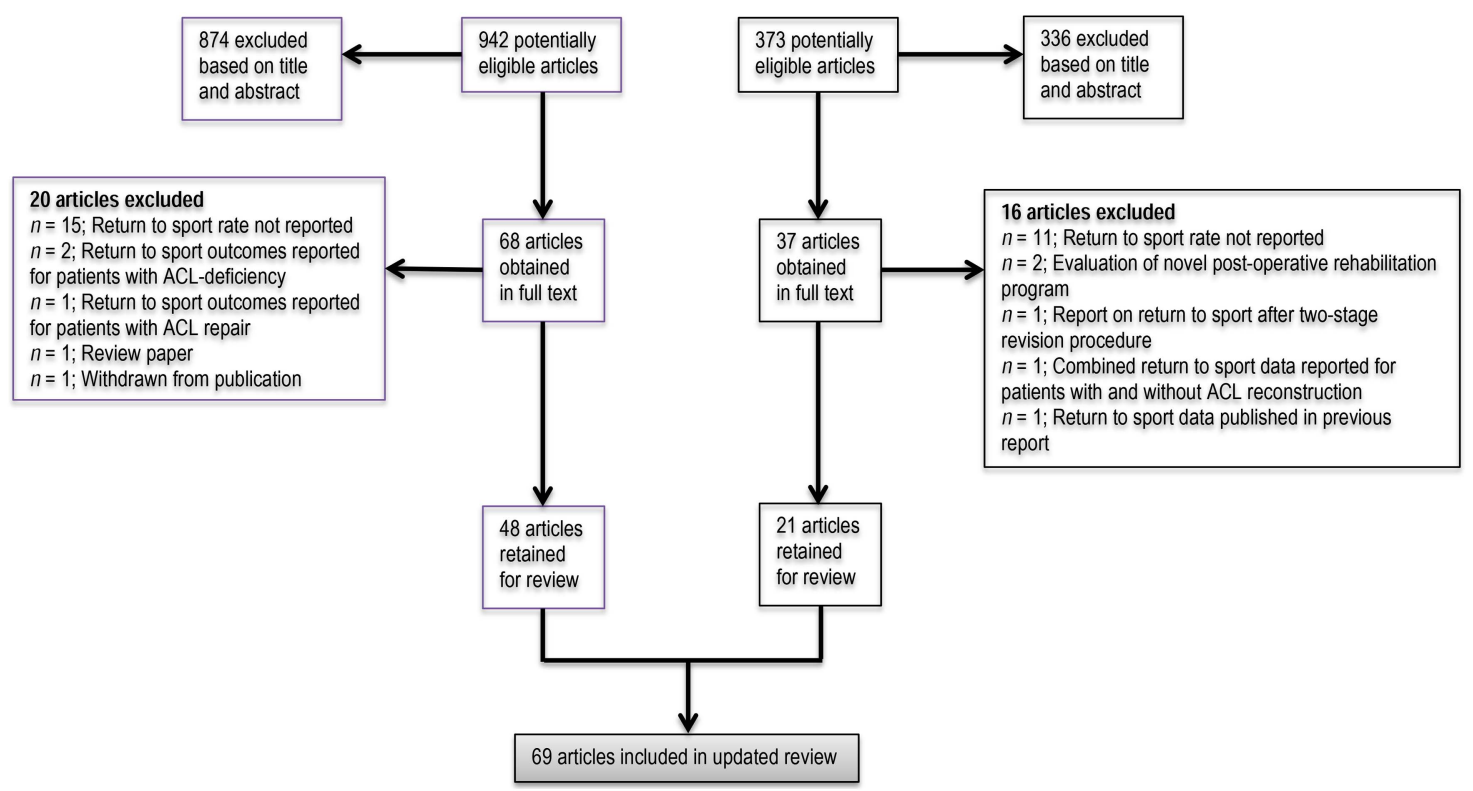

Updated literature search (April 2010 - November 2013)

Figure 1 Article selection ( $\mathrm{ACL}$, anterior cruciate ligament).

of returning to competitive level sport (OR, 95\% CI 2.4, 1.9 to $3.1, \mathrm{p}<0.001)$. On the other hand, pooled data from 34 articles $(n=2647)$ showed that people with patellar tendon grafts had 1.2 times the odds of returning to their preinjury level sport compared with those with hamstring tendon grafts (OR, 95\% CI $1.2,1.1$ to $1.5, p=0.01)$. The proportions of people with hamstring and patellar tendon grafts who returned to sport are shown in table 2 .

\section{Other factors}

Pooled data from 57 articles $(n=6877)$ showed that elite athletes had greater odds of returning to any sport (OR, 95\% CI 1.4, 1.02 to $2.0, \mathrm{p}=0.04)$. Pooled data from 53 articles $(\mathrm{n}=4220)$ showed that elite athletes had more than twice the odds of returning to their preinjury level sport than non-elite athletes (OR, 95\% CI 2.5, 2.0 to 3.1, $\mathrm{p}<0.0001)$. Pooled data from 28 articles $(n=2918)$ showed that elite athletes had approximately six times the odds of returning to competitive sport as non-elite athletes (OR, 95\% CI 5.9, 4.6 to $7.5, \mathrm{p}<0.001)$. The proportions of elite and non-elite athletes who returned to sport are shown in table 2.

The length of follow-up did not influence the return to sport rate for the three rates examined (table 2). The average follow-up was 40 months ( $\mathrm{SD}=28.7$, range $12-156$ months).

\section{DISCUSSION}

We summarised the current evidence regarding return to sport rates following ACL reconstruction surgery. Return to sport outcomes were analysed for over 7000 participants who had undergone ACL reconstruction surgery and, on average, 4 in every 5 returned to some form of sport following surgery. However, the rates of return to the preinjury level and competitive level sport were lower. On average, two in three participants returned to their preinjury level, and only 55\% returned to competitive level sport.

These findings extend the conclusions of our previous review $^{2}$ where, depending on the level of sport (ie, any, preinjury, competitive level sport), the return to sport rate ranged from $44 \%$ to $80 \% .^{1}$ There was a change in the rate of return to competitive level sport in the current review; we now report $55 \%$ compared with $44 \%$ in the previous review.

The addition of five recent studies examining the return to sport rate of elite athletes explains the superior return to competitive sport rate. In the current review, elite athletes have a higher return to sport rate than non-elite athletes. In the $2011^{1}$ meta-analysis, there were only four studies of elite athletes included, whereas in the current review nine studies of elite athletes were included in the return to competitive sport meta-analysis. In the current review, the return to sport rate varied based on certain contextual factors, suggesting that the context within which an individual functions is associated with the prospects for returning to sport after surgery.

\section{Relationship between physical functioning and returning to sport}

This review builds on previous analyses of return to sport rate following ACL reconstruction ${ }^{145}$ by examining whether specific impairments, activities and contextual factors were associated with returning or not returning to participation in the preinjury level sport. A focus of previous research has been on measuring physical knee function after ACL reconstruction surgery. In pooling the results of previous studies of objective physical function, the current review found that people with objective knee function classified as abnormal or severely abnormal were just as likely to return to their preinjury level sport as those with knee function classified as normal or nearly abnormal. However, when those with normal knee function were compared to those with nearly normal, abnormal or severely abnormal knee function, they had approximately twice the odds of returning to their preinjury level sport. There was also a small effect favouring a more symmetrical hopping performance and returning to the preinjury level sport (standardised mean difference 0.3 ).

These findings of an association between better physical function and returning to the preinjury level sport in the current review contradict those of a previous study, published when there were less data available, which did not find a relationship 
Figure 2 Pooled rate of return to any sport $(81 \%, 95 \% \mathrm{Cl} 74 \%$ to $87 \%)$.

\section{Proportion meta-analysis plot [random effects]}

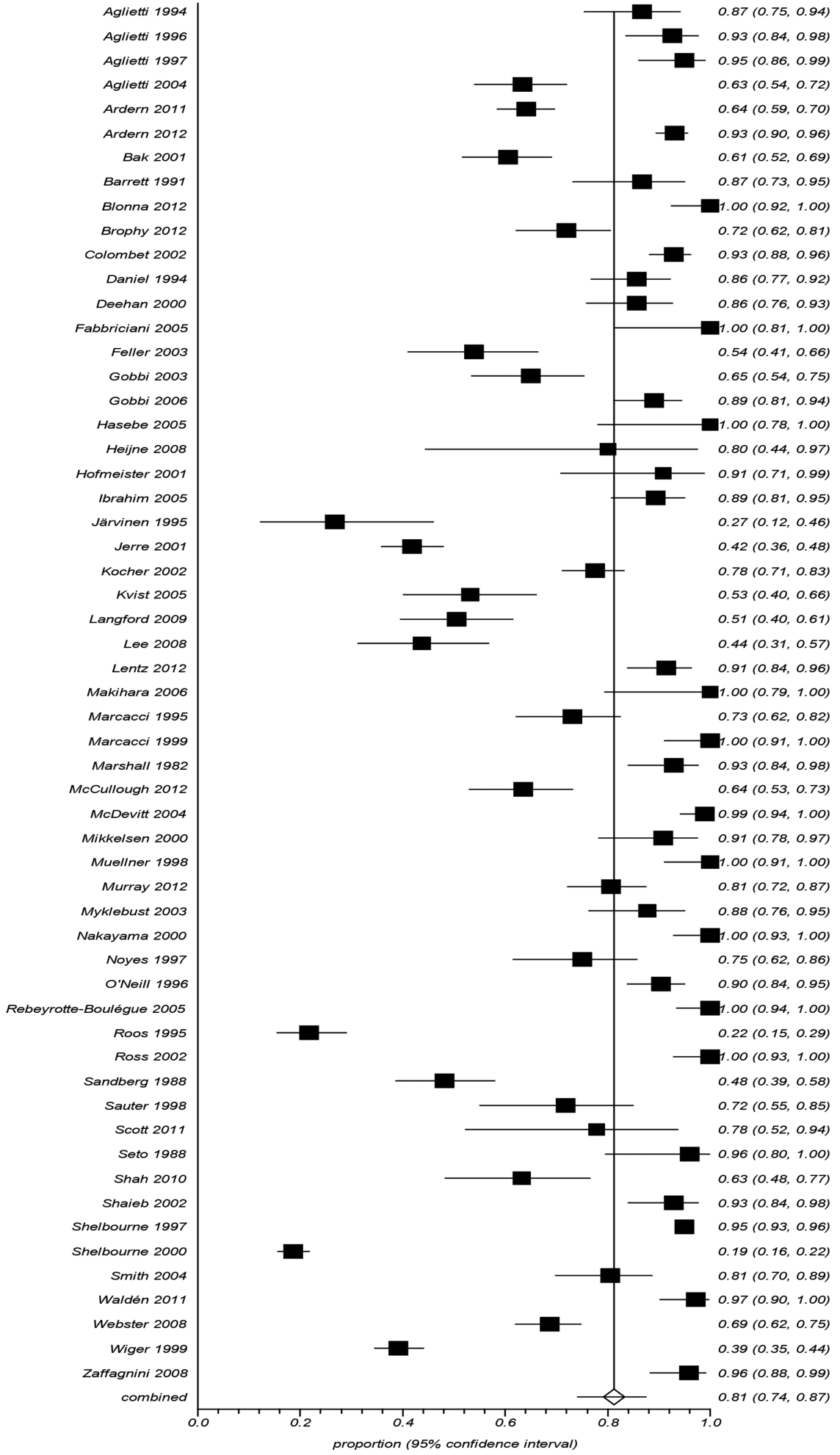

between physical functioning and participation in a number of activities including sport. ${ }^{46}$ However, our findings support the notion that to successfully participate in sport, athletes may need to achieve a certain level of physical functioning to enable optimal performance of sport-specific tasks. ${ }^{47}$ This has clinical implications in terms of the provision of postoperative rehabilitation programmes and clearance of athletes to return to sport. Furthermore, the large effect favouring a higher physical activity level (Marx Activity Scale score) and returning to the preinjury level sport (standardised mean difference 3.1) demonstrates the relationship between more frequent participation in sport-specific activities (jumping, running, cutting, pivoting) and returning to sport.

\section{Gender differences in returning to sport}

Men were approximately one and a half times more likely than women to return to either their previous level of sport 
Figure 3 Pooled rate of return to preinjury level sport $(65 \%, 95 \% \mathrm{Cl}$ $59 \%$ to $72 \%$ ).

\section{Proportion meta-analysis plot [random effects]}

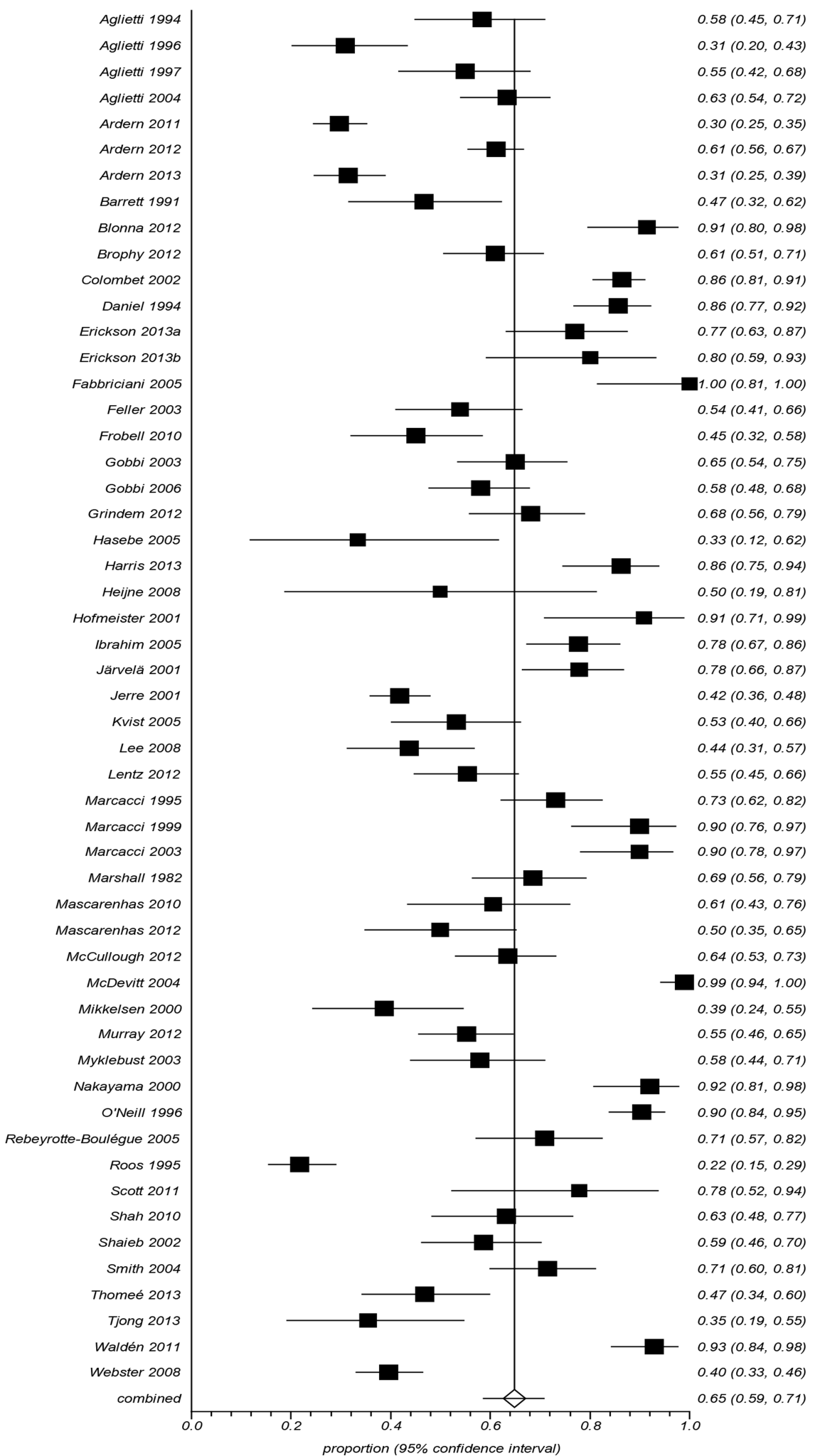

$(\mathrm{OR}=1.4)$ or competitive sport $(\mathrm{OR}=1.7)$. It could be speculated that more men than women returning to competitive sport might reflect differences in motivation for participating in sport, $^{48} 49$ or that men were more likely to play competitive level sport compared with women. Equally, the fact that there was no difference in the rate of return to any sport might suggest that overall a similar number of women and men return to sport following surgery, but that more women participate at a reduced level or intensity. It could also be speculated that gender differences may reflect different social roles. However, empirical evidence is lacking to explain gender differences in returning to sport. 
Figure 4 Pooled rate of return to competitive sport $(55 \%, 95 \% \mathrm{Cl} 46 \%$ to $63 \%)$.
Proportion meta-analysis plot [random effects]

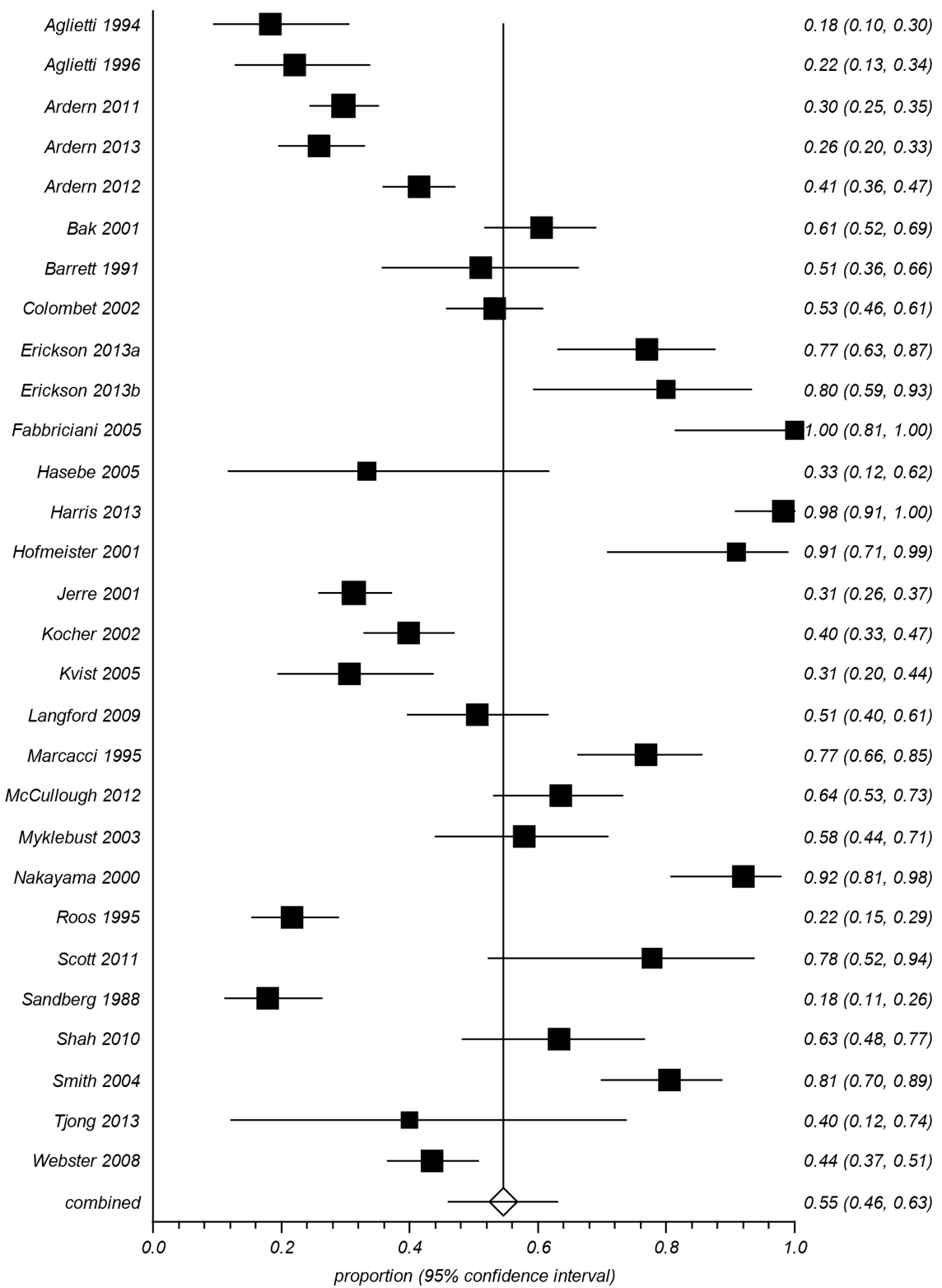

\section{Autograft choice-patellar tendon versus hamstring}

Graft choice for ACL reconstruction surgery continues to be debated. In the current review, people who received patellar tendon grafts had modestly increased odds (1.2 times) of returning to their preinjury level sport compared with those with hamstring tendon grafts. However, this relationship was reversed for returning to competitive level sport, where people who received hamstring tendon grafts had more than twice the odds of returning $(\mathrm{OR}=2.4)$. How can one explain these seemingly contrasting results?

First, although the difference between the two graft types for returning to preinjury sport was statistically significant, the actual percentage difference was only $5 \%$. Second, most of the included studies were not randomised for graft choice, and therefore selection bias is very likely to have confounded the results; many surgeons have a preference for the graft they will use for professional players. ${ }^{50}$ Indeed, there were only five relevant randomised studies, ${ }^{21} 51-54$ and when data from four of these $^{21} 515254 \quad\left(n=288\right.$; the fifth $^{53}$ did not present separate data for graft type, but stated that there was no statistical difference in the rate of return to preinjury level sport between hamstring and patellar tendon grafts) were combined using meta-analysis, there was no difference in the return to preinjury level sport rates between the two grafts (OR, 95\% CI 1.02, 0.7 to $1.5, \mathrm{p}=0.92)$. Also, the combined return rate was slightly higher for hamstring grafts $(59 \%$ hamstring vs $57 \%$ patellar tendon) than for patellar tendon grafts. While there is inconsistency in these results, the results of the randomised studies suggest that any differences in the rate of return to preinjury level sport between graft types may be small.

\section{Graft type and subsequent ACL injury}

In terms of returning to competitive sport, it is relevant to note that there were approximately three times as many hamstring tendon grafts $(n=1164)$ as patellar tendon grafts $(n=394)$ in the meta-analysis. Therefore, the combined return to competitive sport rates will be weighted towards the mean of the hamstring grafts. 


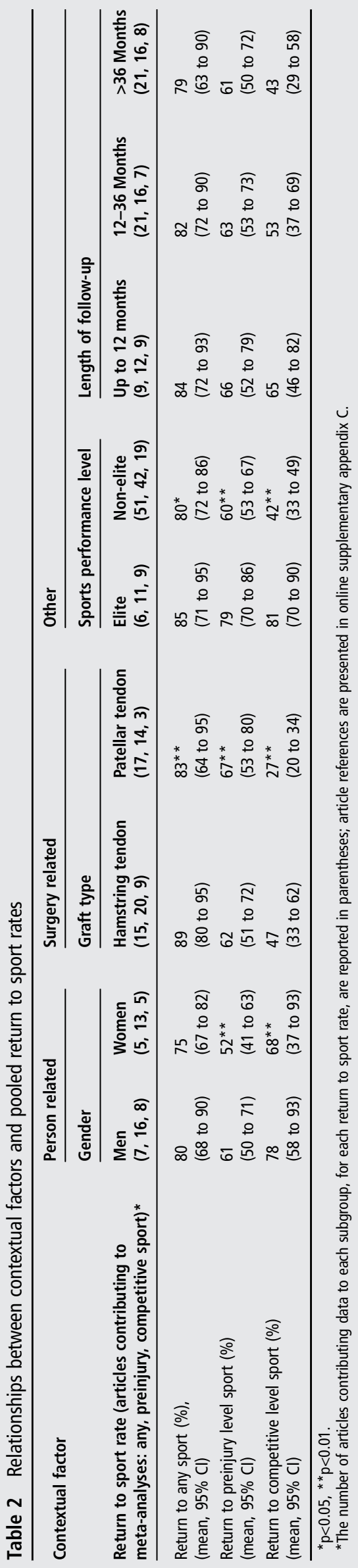

There is conflicting evidence regarding the risk of ACL graft rupture according to graft type. Some recent studies have suggested that there may be an increased risk of ACL graft rupture for people with hamstring grafts. ${ }^{55-57}$ However, other studies have found no relationship between graft type and risk of ACL rupture. ${ }^{42}$ It may be possible that the return to competitive sport rate could be a confounding factor in analyses of ACL graft rupture risk considering our findings of the higher rate of return to competitive sport for hamstring tendon grafts compared with patellar tendon grafts. Conversely, for contralateral ACL injury, in the studies that have found a difference between graft types, a higher incidence has been reported in patients with patellar tendon grafts. ${ }^{58} 59$

The difference in return to competitive sport rate between graft types must be interpreted with caution as the majority of studies were non-randomised, and in the only randomised controlled trial (RCT) to report return to competitive sport rates, which was published approximately 20 years ago, ${ }^{22}$ there was no difference between grafts. It would be premature to make firm conclusions from the data included in the current review as there are limited data from RCTs and few recent studies with patellar tendon grafts.

\section{Contextual factors-considerations for clinicians}

Most of the contextual factors identified in this review as impacting on returning to sport are non-modifiable, for example, gender, age and preinjury sports participation level. In contrast, the fact that physical functioning is modifiable with appropriate intervention may be a reason why the focus of postoperative rehabilitation has been on addressing knee function impairments. ${ }^{456061}$ It seems reasonable to suggest that to be able to cope with the physical demands of playing sport, treatment for ACL injury must promote physical recovery such that an athlete has the physical capacity to play their sport.

Our previous review ${ }^{1}$ demonstrated that ACL reconstruction surgery seems to be moderately effective at restoring knee movement, muscle strength and neuromuscular control. However, to maximise participation outcomes after surgery, clinicians may also need to consider factors in addition to physical functioning.

Large effects favouring positive psychological responses and returning to the preinjury level sport were identified in the current review (standardised mean difference of at least 0.9), which is particularly important because psychological factors may be modifiable with appropriate interventions. The most recently published clinical practice guidelines from 2010, for rehabilitation following ACL reconstruction surgery, do not emphasise interventions for psychological factors. ${ }^{61}$ However, given that the data presented in these published guidelines are, in some cases, over 10 years old, and that more recently published studies have highlighted the potential importance of addressing psychological factors, it is likely that many clinicians may consider psychological factors in their rehabilitation approach.

Incorporating routine screening for psychological responses that could hinder returning to sport into standard postoperative rehabilitation programmes may help clinicians identify athletes at risk of not returning to sport. This may also enable appropriate and timely interventions to be implemented that address psychological factors. One example of an intervention that may have merit for promoting the behaviour of returning to sport following surgery is health coaching, which involves goal setting, motivational interviewing and cognitive-behavioural strategies to bring about behaviour change. ${ }^{39}$ The current review suggests that further research may be warranted to see if 
addressing individually relevant contextual factors during rehabilitation might improve the return to sport rate.

It could be speculated that the level of investment an athlete has in sport may influence the individual's likelihood of returning to sport after ACL injury and surgery. Elite athletes may have greater time invested in sport through longer training hours, higher performance expectations because they compete at an advanced level, and they may derive financial benefit from playing sport either as a professional player or as a sport scholarship holder. Elite players may also have access to intensive and highly structured support from medical and rehabilitation professionals that is not available to non-elite athletes. This may explain why elite athletes had twice the odds of returning to their preinjury level sport, and six times the odds of returning to competitive sport as non-elite athletes.

Younger athletes were also more likely to return to their preinjury level sport (standardised mean difference -0.3). Age may act as a proxy for other factors such as family or employment commitments that limit the time an individual has to participate in sport. It may be reasonable to assume that younger athletes may have fewer family or employment commitments that could mean they have more available time to participate in sport, and that sport may also represent the major social outlet for younger athletes. For those athletes whose lives and social networks are inherently structured around participation in sport, a stronger sense of athletic identity may be a positive motivator for returning to sport. However, empirical data are required to confirm these hypotheses.

Surgery is typically recommended for athletes who wish to return to sport, particularly those that involve cutting and pivoting, following an ACL injury. ${ }^{62}$ However, this review demonstrates that just because an athlete has surgery, it does not automatically mean that they will return to sport. There may be reasons that explain why people do not return to sport, such as poorly performed surgery, poorly performed postoperative rehabilitation, lax criteria for rehabilitation progression and clearance to return to sport, and a lack of emphasis on addressing psychological factors in rehabilitation programmes. It is also important to note that there may be other reasons that surgery is recommended, for example, employment in a physically demanding job, or recurrent knee instability with activities of daily living. On the other hand, the fact that a proportion of athletes who have ACL reconstruction surgery do not return to sport, despite this being one of the main motivators for choosing surgery in the first place, could suggest that the desire to return to sport alone may be an insufficient indication for ACL reconstruction in some cases. A recent randomised clinical trial found no difference in knee function, health status and rate of return to preinjury activity level (including sport) at 2 and 5 years follow-up in young, previously active people, regardless of whether they had operative or non-operative treatment for ACL injury. ${ }^{17} 18$ This preliminary evidence may suggest that some people who do not have surgery may have the potential to achieve similar function and participation outcomes as those who do have surgery.

\section{Strengths and limitations}

The strengths of this review are that it combines data on returning to sport from over 7000 patients after ACL reconstruction surgery, and is the first to present results on the association between contextual factors and returning to sport after surgery. These findings support the emerging body of literature suggesting that recovery of physical function alone may not be all that is required for successful return to sport following ACL reconstruction. ${ }^{63}$

One limitation of the current review is that the meta-analysis model did not allow for the investigation of interaction effects between physical and contextual factors. Hence, it is not possible to make conclusions regarding whether particular combinations of factors contribute to improving or reducing the chances of an individual returning to sport after surgery. The secondary aim of this review was also limited by the fact that of the 66 included articles, only 7 reported separate physical functioning data, 2 reported separate data on age and 4 reported separate psychological factors data for participants who did and did not return to sport. If the relationship between these factors and returning to sport is reported more consistently in future studies, it will facilitate more definitive conclusions being made regarding the impact of aspects of physical functioning and contextual factors on participation after surgery.

The high $\mathrm{I}^{2}$ values indicated that there was a large amount of heterogeneity in the return to sport data. Heterogeneity, measured by the $\mathrm{I}^{2}$ statistic, provides an indication of the level of agreement or disagreement in return to sport rates between included articles. ${ }^{14}$ This is most easily observed in the forest plots, which show the large range of return to sport rates reported across individual studies. The heterogeneity may arise due to differences in sampling across different studies; and while some studies reported return to sport as a primary outcome, in the majority of studies, evaluating returning to sport was not the primary focus of the study. Higher heterogeneity reduces the certainty of the mean return to sport rate. However, the use of a random-effects meta-analysis model (as in the current review) takes the high heterogeneity into account, providing a more conservative estimate of the return to sport rate (a higher proportion returning to sport).

Specific contextual factors within individual studies, which could not be accounted for in the meta-analysis, may have contributed to variability in the return to sport rates. To be included for review, studies must have reported a return to sport rate. This means that studies evaluating return to sport using only an activity rating scale such as the Tegner Activity Scale or IKDC sports activity scale were not included. Therefore, there may be additional information about returning to sport that was not considered in the current review.

A final limitation is the quality of the articles included for review. For example, item 3 on the quality assessment was fulfilled by the lowest number of articles (64\%). This demonstrated that only $64 \%$ of included articles reported the preinjury participation level of athletes. The preinjury participation level may help to provide an indication of the most appropriate return to sport outcome. For example, if athletes were not playing competitive level sport before their injury, it may not be reasonable to report the return to competitive sport rate.

\section{Clinical conclusion}

Combined data from over 7000 participants provide data to a field that has seen a great deal of personal opinion and conjecture. ${ }^{64}$ We demonstrated that $81 \%$ of people returned to some form of sport after surgery, $65 \%$ returned to their previous level of sport and $55 \%$ returned to competitive sport. Younger age favoured returning to the preinjury level sport. Men had greater odds of returning to their preinjury level sport than women; and elite athletes had greater odds of returning to sport than non-elite athletes. Receiving a hamstring tendon autograft 
favoured returning to competitive level sport, whereas receiving a patellar tendon autograft favoured returning to the preinjury level sport. A positive psychological response favoured returning to the preinjury level sport. Symmetrical knee function also favoured returning to the preinjury level of sport.

\section{Perspectives}

When evaluating sport participation outcomes at the population level, first, it is important to specify the level of participation. In this review, we classified return to sport rates as returning to any sport, the preinjury level sport and competitive level sport. However, it is also important to consider that after surgery some athletes may change sports, change priorities regarding participation in sport or return to participation at a lower level than the preinjury level, and still be satisfied with their participation outcomes. Therefore, determinants of success in returning to sport should ideally be individualised according to the goals of the patient.

Most of the contextual factors that impacted on returning to sport in the current review were non-modifiable. However, positive psychological responses, and subjective and objective measures of physical functioning, which are potentially modifiable with appropriate interventions, favoured returning to the preinjury level sport. This may suggest that specifically addressing both psychological factors and physical functioning factors in postoperative rehabilitation programmes is warranted. Future research should examine ways to provide patients with access to rehabilitation programmes that address both psychological factors and physical functioning factors.

\section{What are the new findings?}

- Fifty-five per cent of athletes return to competitive sport after anterior cruciate ligament reconstruction surgery.

- Eighty-one per cent of people return to some form of sport, and $65 \%$ return to their preinjury level sport.

- Younger age, male gender, playing elite sport and having a positive psychological response were contextual factors that favoured returning to the preinjury level sport.

- Symmetrical hopping performance favoured returning to the preinjury level sport.

- People who received hamstring tendon autografts had greater odds of returning to competitive level sport, whereas people who received patellar tendon autografts had greater odds of returning to their preinjury level sport.

Contributors All authors contributed to the conception and design of the review. CLA applied the search strategy, extracted and analysed the data, and wrote the manuscript. CLA and NFT applied the inclusion criteria. CLA and KEW completed the assessment of risk of bias. All authors contributed to the interpretation of data. NFT, JAF and KEW critically revised the manuscript for important intellectual content. CLA is responsible for the overall content as guarantor. All authors approved the final version.

Competing interests None.

Provenance and peer review Not commissioned; externally peer reviewed.

\section{REFERENCES}

1 Ardern $\mathrm{CL}$, Webster $\mathrm{KE}$, Taylor NF, et al. Return to sport following anterior cruciate ligament reconstruction surgery: a systematic review and meta-analysis of the state of play. Br J Sports Med 2011;45:596-606.

2 Heijne $A$, Axelsson $\mathrm{K}$, Werner $\mathrm{S}$, et al. Rehabilitation and recovery after anterio cruciate ligament reconstruction: patients' experiences. Scand J Med Sci Sports $2008 \cdot 18 \cdot 325-35$
3 Kvist J, Ek A, Sporrstedt K, et al. Fear of re-injury: a hindrance for returning to sports after anterior cruciate ligament reconstruction. Knee Surg Sports Traumatol Arthrosc 2005;13:393-7.

4 Langford JL, Webster KE, Feller JA. A prospective longitudinal study to assess psychological changes following anterior cruciate ligament reconstruction surgery. $\mathrm{Br}$ J Sports Med 2009;5:377-81.

5 Nakayama Y, Shirai Y, Narita T, et al. Knee functions and a return to sports activity in competitive athletes following anterior cruciate ligament reconstruction. J Nippon Med Sch 2000;67:172-6.

6 Smith FW, Roselund EA, Aune AK, et al. Subjective functional assessments and the return to competitive sport after anterior cruciate ligament reconstruction. $\mathrm{Br} J$ Sports Med 2004:38:279-84.

7 Webster K, Feller J, Lambros C. Development and preliminary validation of a scale to measure the psychological impact of returning to sport following anterior cruciate ligament reconstruction surgery. Phys Ther Sport 2008;9:9-15.

8 World Health Organization. Towards a common language for functioning, disability, and health: the International Classification of Functioning, Disability, and Health. Geneva: World Health Organization, 2002.

9 Centre for Reviews and Dissemination. The process of quality assessment in systematic reviews. Systematic reviews: CRD's guidance for undertaking reviews in health care: Centre for Reviews and Dissemination, 2009.

10 Khan K, ter Riet G, Popay J, et al. Conducting the review: study quality assessment. Undertaking systematic reviews of research on effectiveness: CRD's guidance for those carrying out or commissioning reviews Centre for Reviews and Dissemination, 2001.

11 Downs SH, Black N. The feasibility of creating a checklist for the assessment of the methodological quality both of randomised and non-randomised studies of health care interventions. J Epidemiol Community Health 1998;52:377-84.

12 Slim K, Nini E, Forestier D, et al. Methodological index for non-randomized studies (MINORS): development and validation of a new instrument. ANZ J Surg 2003:73:712-16.

13 Cohen J. Statistical power analysis for the behavioral sciences. 2nd edn. Hillsdale, NJ: Erlbaum, 1988.

14 Higgins JP, Thompson SG, Deeks JJ, et al. Measuring inconsistency in meta-analyses. BMJ 2003;327:557-60.

15 Ardern CL, Taylor NF, Feller JA, et al. Return-to-sport outcomes at 2 to 7 years after anterior cruciate ligament reconstruction surgery. Am J Sports Med 2012:40:41-8

16 Ardern $\mathrm{CL}$, Webster KE, Taylor NF, et al. Return to the preinjury level of competitive sport after anterior cruciate ligament reconstruction surgery: two-thirds of patients have not returned by 12 months after surgery. Am J Sports Med 2011;39:538-43.

17 Frobell RB, Roos EM, Roos HP, et al. A randomized trial of treatment for acute anterior cruciate ligament tears. N Engl J Med 2010;363:331-42.

18 Frobell RB, Roos HP, Roos EM, et al. Treatment for acute anterior cruciate ligament tear: five year outcome of randomised trial. BMJ 2013;346:f232.

19 Brophy R, Schmitz L, Wright R, et al. Return to play and future ACL injury risk after $A C L$ reconstruction in soccer athletes from the Multicenter Orthopaedic Outcomes Network (MOON) Group. Am J Sports Med 2012;40:2517-22.

20 Deehan DJ, Salmon LJ, Webb VJ, et al. Endoscopic reconstruction of the anterior cruciate ligament with an ipsilateral patellar tendon autograft. A prospective longitudinal five-year study. J Bone Joint Surg Br 2000;82:984-91.

21 Aglietti P, Buzzi R, Zaccherotti G, et al. Patellar tendon versus doubled semitendinosus and gracilis tendons for anterior cruciate ligament reconstruction. Am J Sports Med 1994;22:211-18.

22 Daniel DM, Stone ML, Dobson BE, et al. Fate of the ACL-injured patient: a prospective outcome study. Am J Sports Med 1994;22:632-44.

23 McDevitt ER, Taylor DC, Miller MD, et al. Functional bracing after anterior cruciate ligament reconstruction: a prospective, randomized, multicenter study. Am J Sports Med 2004;32:1887-92.

24 Murray JR, Lindh AM, Hogan NA, et al. Does anterior cruciate ligament reconstruction lead to degenerative disease? Thirteen-year results after bone-patellar tendon-bone autograft. Am J Sports Med 2012;40:404-13.

25 Shah VM, Andrews JR, Fleisig GS, et al. Return to play after anterior cruciate ligament reconstruction in National Football League athletes. Am J Sports Med 2010;38:2233-9.

26 Järvinen $\mathrm{M}$, Natri $\mathrm{A}$, Lehto $\mathrm{M}$, et al. Reconstruction of chronic anterior cruciate ligament insufficiency in athletes using a bone-patellar tendon-bone autograft: a two-year follow up study. Int Orthop 1995;19:1-6.

27 Jerre $\mathrm{R}$, Ejerhed $\mathrm{L}$, Wallmon $\mathrm{A}$, et al. Functional outcome of anterior cruciate ligament reconstruction in recreational and competitive athletes. Scand J Med Sci Sports 2001;11:342-6.

28 McCullough KA, Phelps KD, Spindler KP, et al. Return to high school- and college-level football after anterior cruciate ligament reconstruction: a Multicenter Orthopaedic Outcomes Network (MOON) cohort study. Am J Sports Med 2012;40: 2523-9.

29 Myklebust G, Holm I, Maehlum S, et al. Clinical, functional and radiologic outcome in team handball players 6 to 11 years after anterior cruciate ligament injury: a follow up study. Am J Sports Med 2003:31:981-9. 
30 Roos $\mathrm{H}$, Ornell $\mathrm{M}$, Gardsell P, et al. Soccer after anterior cruciate ligament injury: an incompatible combination? Acta Orthop Scand 1995;66:107-12.

31 Erickson BJ, Harris JD, Cvetanovich GL, et al. Performance and return to sport after anterior cruciate ligament reconstruction in male Major League Soccer players. Orthop J Sports Med 2013:1:2325967113497189.

32 Erickson BJ, Harris JD, Fillingham YA, et al. Performance and return to sport after anterior cruciate ligament reconstruction in X-Games skiers and snowboarders. Orthop J Sports Med 2013;1:2325967113511196.

33 Harris JD, Erickson BJ, Bach BR, et al. Return-to-sport and performance after anterior cruciate ligament reconstruction in National Basketball Association players. Sports Health 2013;5:562-8.

34 Lentz TA, Zeppieri G Jr, Tillman SM, et al. Return to preinjury sports participation following anterior cruciate ligament reconstruction: contributions of demographic, knee impairment, and self-report measures. J Orthop Phys Ther 2012;42: 893-901.

35 Scott K, Namdari S, Milby A, et al. Athletic performance after ACL reconstruction in Women's National Basketball Association. Phys Sportsmed 2011;39:36-41.

36 Seto JL, Orofino AS, Morrissey MC, et al. Assessment of quadriceps/hamstring strength, knee ligament stability, functional and sports activity levels five years after anterior cruciate ligament reconstruction. Am J Sports Med 1988;16:170-80.

37 Tjong VK, Murnaghan ML, Nyhof-Young JM, et al. A qualitative investigation of the decision to return to sport after anterior cruciate ligament reconstruction: to play or not to play. Am J Sports Med 2014:42:336-42.

38 Aglietti P, Buzzi R, Menchetti PM, et al. Arthroscopically assisted semitendinosus and gracilis tendon graft in reconstruction for acute anterior cruciate ligament injuries in athletes. Am J Sports Med 1996;24:726-30.

39 Ardern CL, Taylor NF, Feller JA, et al. Psychological responses matter in returning to preinjury level of sport after anterior cruciate ligament reconstruction surgery. Am J Sports Med 2013;41:1549-58

40 Noyes FR, Barber-Westin SD. Anterior cruciate ligament reconstruction with autogenous patellar tendon graft in patients with articular cartilage damage. Am J Sports Med 1997;25:626-34.

41 O'Neill DB. Arthroscopically assisted reconstruction of the anterior cruciate ligament. A prospective randomized analysis of three techniques. J Bone Joint Surg Am 1996;78-A:803-13

42 Webster KE, Feller JA, Leigh WB, et al. Younger patients are at increased risk for graft rupture and contralateral injury after anterior cruciate ligament reconstruction. Am J Sports Med 2014;43:641-7.

43 Lee DY, Karim SA, Chang HC. Return to sports after anterior cruciate ligament reconstruction: a review of patients with minimum 5-year follow-up. Ann Acad Med Singapore 2008;37:273-8.

44 Gobbi A, Francisco R. Factors affecting return to sports after anterior cruciate ligament reconstruction with patellar tendon and hamstring graft: a prospective clinical evaluation. Knee Surg Sports Traumatol Arthrosc 2006;14:1021-8.

45 Kvist J. Rehabilitation following anterior cruciate ligament injury: current recommendations for sports participation. Sports Med 2004;34:269-80.

46 Ross MD, Irrgang JJ, Denegar CR, et al. The relationship between participation restrictions and selected clinical measures following anterior cruciate ligament reconstruction. Knee Surg Sports Traumatol Arthrosc 2002;10:10-19.

47 Ericsson $\mathrm{YB}$, Roos EM, Frobell RB. Lower extremity performance following $\mathrm{ACL}$ rehabilitation in the KANON-trial: impact of reconstruction and predictive value at 2 and 5 years. Br I Sports Med 2013;47:980-5.
48 Deaner RO, Geary DC, Puts DA, et al. A sex difference in the predisposition for physical competition: males play sports much more than females even in the comtemporary U.S. PLOS ONE 2012;7:e49168.

49 Kondric M, Sindik J, Furjan-Mandic G, et al. Participation motivation and students' physical activity among sports students in three countries. I Sports Sci Med 2013:12:10-18.

50 Poulsen MR, Johnson DL. Graft selection in anterior cruciate ligament surgery. Orthopedics 2010;33:832-5

51 Aglietti $\mathrm{P}$, Giron $\mathrm{F}$, Buzzi $\mathrm{R}$, et al. Anterior cruciate ligament reconstruction: bone-patellar tendon-bone compared with double semitendinosus and gracilis tendon grafts-a prospective, randomized clinical trial. J Bone Joint Surg Am 2004;86:2143-55.

52 Feller JA, Webster KE. A randomized comparison of patellar tendon and hamstring tendon anterior cruciate ligament reconstruction. Am I Sports Med 2003;31:564-73.

53 Ibrahim SA, Al-Kussary IM, Al-Misfer AR, et al. Clinical evaluation of arthroscopically assisted anterior cruciate ligament reconstruction: patellar tendon versus gracilis and semitendinosus autograft. Arthroscopy 2005; 21:412-17.

54 Shaieb MD, Kan DM, Chang SK, et al. A prospective randomized comparison of patellar tendon versus semitendinosus and gracilis tendon autografts for anterior cruciate ligament reconstruction. Am I Sports Med 2002;30:214-20.

55 Barrett DS, MacKenney RP. Maclntosh-Jones reconstruction for the unstable knee. Injury 1991;22:282-6.

56 Maletis GB, Inacio MCS, Desmond JL, et al. Reconstruction of the anterior cruciate ligament. Association of graft choice with increased risk of early revision. Bone Joint J 2013;95-B:623-8.

57 Persson A, Fjeldsgaard K, Gjertsen J, et al. Increased risk of revision with hamstring tendon grafts compared with patellar tendon grafts after anterior cruciate ligament reconstruction. A study of 12,643 patients from the Norwegian Cruciate Ligament Registry, 2004-2012. Am J Sports Med 2014;42:285-91.

58 Pinczewski LA, Lyman J, Salmon LJ, et al. A 10-year comparison of anterior cruciate ligament reconstructions with hamstring tendon and patellar tendon autograft: a controlled, prospective trial. Am J Sports Med 2007;35:564-74.

59 Leys T, Salmon $\mathrm{L}$, Waller $A$, et al. Clinical results and risk factors for reinjury 15 years after anterior cruciate ligament reconstruction: a prospective study of hamstring and patellar tendon grafts. Am J Sports Med 2012; 40:595-605.

60 Adams D, Logerstedt D, Hunter-Giordano A, et al. Current concepts for anterior cruciate ligament reconstruction: a criterion-based rehabilitation progression. J Orthop Sports Phys Ther 2012;42:601-14.

61 van Grinsven S, van Cingel RE, Holla CJ, et al. Evidence-based rehabilitation following anterior cruciate ligament reconstruction. Knee Surg Sports Traumatol Arthrosc 2010;18:1128-44.

62 Myklebust $\mathrm{G}$, Bahr R. Return to play guidelines after anterior cruciate ligament surgery. Br J Sports Med 2005;39:127-31.

63 Ardern CL, Taylor NF, Feller JA, et al. A systematic review of the psychological factors associated with returning to sport following injury. $\mathrm{Br}$ I Sports Med 2013:47:1120-6.

64 Renström P. Eight clinical conundrums relating to anterior cruciate ligament (ACL) injury in sport: recent evidence and a personal reflection. Br I Sports Med 2013:47:367-72. 